Bibliotecas. Vol 36, N¹ Especial, 2018. EISSN: 1659-3286

URL: http://www.revistas.una.ac.cr/index.php/bibliotecas/index

DOI: http://dx.doi.org/10.15359/rb.36-1.1

Licencia: Creative Commons (BY-NC-ND) 4.0 Internacional

\title{
Aproximación al perfil del docente del grado en Información y documentación de la Universidad Complutense de Madrid
}

\section{Approach to the Profile of Teaching Degree in Information and Documentation from the Complutense University of Madrid}

Carlos Miguel Tejada-Artigas* Facultad de Ciencias de la Documentación, Universidad Complutense de Madrid

\section{Resumen}

Se estudian las características del profesorado del grado en Información y documentación de la Universidad Complutense de Madrid tales como su número, su evolución, su distribución por categorías, su uso de las tecnologías de la información, su pertenencia a grupos de investigación y sus publicaciones.

Palabras clave: Profesores universitarios, perfil, Universidad Complutense de Madrid, España.

\section{Abstract}

There are studied the characteristics of the professorship of the grade in Information and documentation of the Complutense University of Madrid, such as: his number, his evolution, his distribution for categories, his use of the information technologies, his ownership to groups of research and his publications.

Keywords: Professorship, Profile, Complutense University of Madrid, Spain. 


\section{Introducción}

La universidad española, desde hace veinte años, vive inmersa en un proceso de debate y cambio motivado por su integración en el Espacio Europeo de Educación Superior (EEES). Han sido años de cambios legislativos, pero sobre todo de una reflexión acerca del sentido de la universidad en la sociedad y la calidad de la enseñanza que ofrecemos.

El origen de este espacio lo podemos encontrar en la Declaración de La Sorbona ( 25 de mayo de 1998) ${ }^{1}$ que fue firmada por los Ministros de Educación de Francia, Alemania, Italia y Reino Unido y se le considera el comienzo del proceso de convergencia ya que señala la necesidad de "crear un espacio europeo de educación superior".

Pero, en realidad el documento clave del proceso, hasta tal punto de que en ocasiones se denomina "proceso de convergencia" en lugar del "proceso de Bolonia", es la Declaración de Bolonia (19 de junio de 1999) ${ }^{2}$. Fue firmada por los ministros de educación de veintinueve estados europeos y es la encargada de fijar los elementos fundamentales para la construcción del "Espacio Europeo de Educación Superior". Los temas más importantes en los que se debe basar esta convergencia europea son los siguientes:

- La estructura de titulaciones. se debe adoptar un sistema fácilmente legible y comparable de titulaciones al servicio de una mayor transparencia en el mercado. Este sistema de titulaciones tendrá dos niveles principales: grado y postgrado. Además, se introduce un suplemento europeo al título.

- El sistema de créditos: se crea el sistema ECTS (European Credit Transfer System) para fomentar la comparabilidad de las titulaciones y promover la movilidad de estudiantes y titulados.

- La movilidad de estudiantes, profesores y personal administrativo.

- La cooperación europea para garantizar la calidad.

- La dimensión europea en la educación superior, sobre todo en lo referente al desarrollo curricular, la cooperación institucional, los esquemas de movilidad y los programas integrados de estudios, formación e investigación. 
Pero incomprensiblemente, en los documentos políticos y normativos del proceso de Bolonia apenas se señala el papel del profesorado tal y como se explicará más adelante.

\section{Objetivo y metodología utilizada}

El objetivo del presente trabajo es trazar una panorámica de las características del profesorado del grado en Información y documentación que se imparte en la Facultad de Ciencias de la Documentación de la Universidad Complutense de Madrid (UCM). La metodología utilizada ha sido bibliográfica, recopilando y analizando documentos que tienen que ver con el objetivo propuesto. Mucha documentación utilizada no está publicada por los canales habituales por lo que se consideraría literatura gris.

\section{Resultados}

\section{a. El profesorado y el EEES}

La sensación, casi 20 años después de la aparición del Proceso de Bolonia, es que ha sido más bien un proceso político más que educativo, en el sentido de que no ha habido una transformación radical de la docencia, tal como se pretendía. Como se ha señalado en la introducción, hay muy poca normativa en la que aparezca la figura del profesor, que es la clave de cualquier reforma universitaria. Nos encontramos ante una definición de su nuevo perfil cuando se precisa el Sistema Europeo de Transferencia de Créditos (ECTS): "basado en el aprendizaje y centrado en el alumno". Al docente se le demanda "que guíe/acompañe al alumno/a a través de un conjunto de actividades educativas donde la clase presencial es un elemento para la consecución de una serie de competencias en las que los conocimientos (su comprensión y su manejo) son una parte" (ANECA, 2003, p. 15).

En el año 2006, un equipo de investigadores liderados por el profesor Valcárcel presentaba el informe final de una investigación que hacía un diagnóstico sobre la implantación del EEES basado en una encuesta a los responsables universitarios de la Convergencia Europea. Se 
recogen algunas conclusiones que afectan al profesorado y que a la fecha actual no han perdido vigencia:

- La escasa inversión económica para soportar un cambio de gran envergadura que implica el proceso de transición y que debería aplicarse a diversos "nichos": desde el cambio/mejora de infraestructuras docentes hasta la formación adecuada e incentivación del profesorado y PAS.

- La docencia no es una actividad del profesorado plenamente reconocida y valorada.

- La dedicación del profesorado a la renovación es imprescindible para alcanzar el éxito en el proceso.

- No se ha emprendido, salvo excepciones, una reforma integral y sistemática de las metodologías docentes en las instituciones universitarias. Muchas medidas en este sentido han sido aisladas e inconexas, escasamente preparadas, poco profundas, coyunturales y planteadas sin convicción más para "cumplir" que para realmente cambiar (Valcárcel, 2006, p. 75).

Así pues, a partir de la propia experiencia personal del proceso vivido por el autor y de la revisión de diferentes estudios al respecto, se puede afirmar que en la actualidad, con respecto al profesorado, sigue habiendo cuatro problemas que no ha solucionado el EEES en España: su formación, dedicación, evaluación e incentivación.

La docencia del profesor es un proceso cada vez más complejo en el que son necesarias distintas competencias, tal como las identifica el profesor Valcárcel (2004):

- Competencias cognitivas sobre su disciplina que faciliten el aprendizaje de su alumnado.

- Competencias metacognitivas que le conviertan en un profesional reflexivo y autocrítico con su enseñanza para mejorarla.

- Competencias comunicativas o dominio de los lenguajes científicos y de sus diferentes registros (artículos, informes, ensayos, conferencias, lecciones...).

- Competencias gerenciales de la enseñanza (utilización de recursos en diversos ambientes y entornos de aprendizaje).

- Competencias sociales que le permitan el liderazgo, la cooperación, el trabajo en equipo. 
- Competencias afectivas que aseguren una docencia responsable y comprometida con la formación de su alumnado.

Lamentablemente, tal como afirma la profesora Madrid (2005):

En la universidad española no ha existido una tradición o una cultura institucional que considerase fundamental la formación docente de su profesorado. Casi quedaba sobreentendido que, con la experiencia del profesor (tanto en su etapa de alumno como por el ejercicio mismo de la enseñanza), bastaba para ser considerado un buen profesional, un buen comunicador-transmisor del conocimiento. (p.59)

Aunque es verdad que en estos últimos años muchas universidades han desarrollado planes de formación del profesorado con cursos innovadores sobre inteligencia emocional, solución de conflictos o atención a la diversidad, el porcentaje de profesores que los lleva a cabo sigue siendo bastante bajo.

El segundo problema que se identificaba es que sigue sin haber una dedicación docente clara. A pesar de que el proceso de Bolonia conlleva un nuevo tipo de formación, basada en el aprendizaje del alumno, la dedicación docente se sigue midiendo por las clases magistrales, es decir, el número de horas de permanencia en el aula.

El tercer y cuarto problema, la evaluación e incentivación, tal como señala el profesor Valcárcel (2006) están unidos y se hace necesaria:

Revitalizar la importancia de las actividades docentes en las universidades y soslayar la creencia bastante generalizada entre el profesorado de que dedicarse a esta actividad es sólo para cumplir, pero que es una pérdida de tiempo a efectos de promoción y reconocimiento. Es preciso un enfoque integral basado en el triángulo formaciónevaluación-reconocimiento/incentivación. Este nuevo enfoque es imprescindible para que el proceso de transición hacia el EEES sea un éxito. (p. 76) 
En la evaluación y la asignación de inventivos económicos del profesorado sigue primando su actividad investigadora frente a la docente y a la de gestión universitaria. Ello conlleva que los profesores estén más preocupados en publicar -no en investigar- en revistas de impacto que en desarrollar una docencia de calidad e innovadora. Así pues, tal como señalan los profesores Alfaro y Pérez (2011)

El poco reconocimiento de la docencia es uno de los mayores inconvenientes a la hora de realizar un cambio en las costumbres docentes de los profesores. Adicionalmente, la innovación docente tampoco es suficientemente reconocida en la carrera profesional, a pesar de los tímidos esfuerzos que se realizan por valorarla desde las propias universidades y desde las agencias responsables de la acreditación, evaluación y contratación del profesorado. La incorporación al EEES supone, entre otras cuestiones, un cambio profundo en las prácticas docentes del profesorado universitario, cambio que, paradójicamente, no es valorado ni reconocido social e institucionalmente. (p.42)

Se hace, pues necesario armonizar las diferentes facetas del profesor universitario y "crear una cultura docente responsable, prestigiada y comprometida con las buenas prácticas, con la satisfacción profesional" (Madrid, 2005, p.61).

\section{b. Perfil del profesorado del grado en Información y documentación de la UCM}

\section{b.1 Desarrollo histórico}

Las titulaciones universitarias oficiales en Biblioteconomía y Documentación en la Universidad Complutense de Madrid comienzan en el curso 1990-1991 con la impartición de la Diplomatura en Biblioteconomía y Documentación en la Escuela Universitaria de Biblioteconomía y Documentación. Estos estudios de primer ciclo duraban tres años. Los estudios de segundo ciclo, la Licenciatura en Documentación, comenzaron a impartirse en la UCM en el curso 1996-1997 en la Facultad de Ciencias de la Información y tenían una duración de dos años. En 2006 se creó la Facultad de Ciencias de la Documentación para impartir las dos titulaciones señaladas. 
El EEES, tal como se ha señalado, supone un cambio en la estructura de titulaciones y a partir de ahora habrá un grado de duración de cuatro años y un postgrado -un máster- de uno. Además, se adopta una nueva denominación: Grado en Información y Documentación ${ }^{3}$ y se empieza a impartir en el curso 2009-2010 en la Facultad de Ciencias de la Documentación.

\section{b.2 La evolución del número de alumnos de la titulación}

Los datos de matrícula de primer curso en la Universidad Complutense de Madrid en los estudios de nuestra área (Diplomatura en biblioteconomía y documentación: curso 1992-93 a curso 2008-2009 y Grado en Información y Documentación: curso 2009-2010 a curso 2012 a 2013) han tenido una oscilación importante, tal como se observa en el Gráfico 1.

\section{Gráfico 1}

\section{Alumnos de nuevo ingreso UCM}

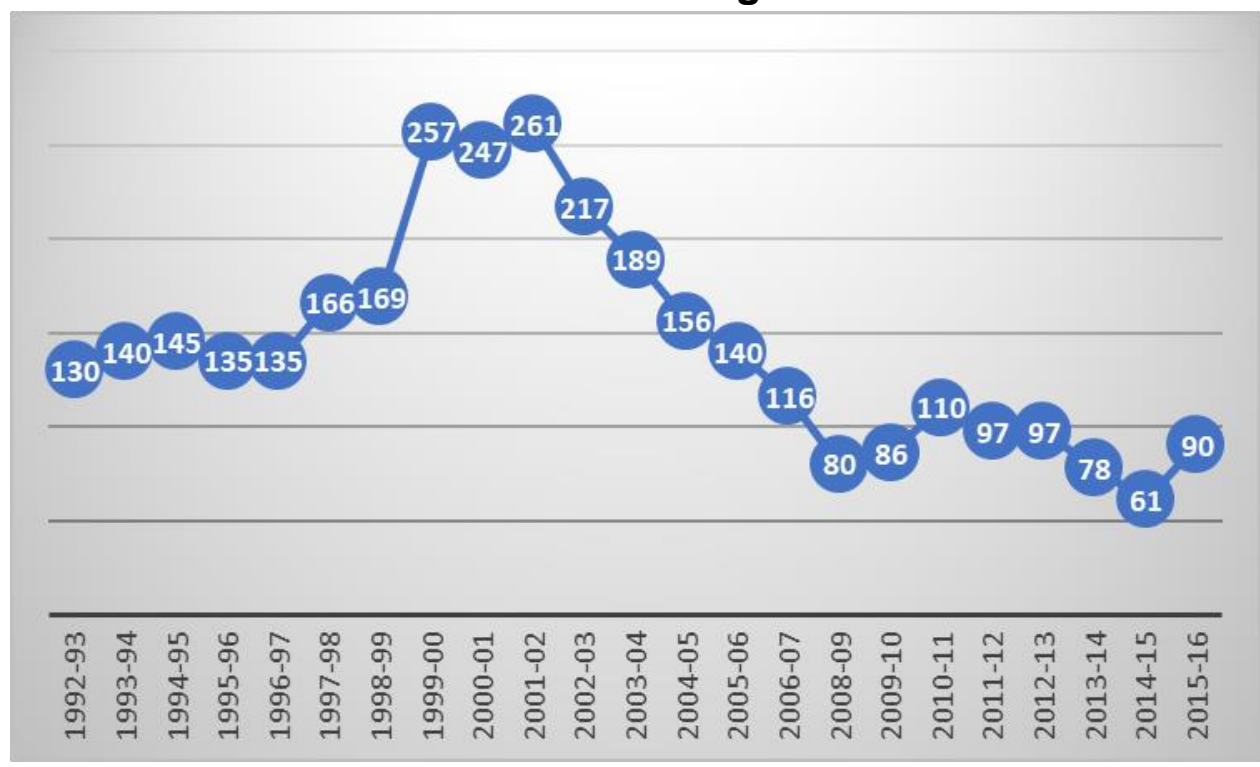

En las titulaciones de Diplomatura en biblioteconomía y documentación (curso 1992-93 a curso 20082009) y de Grado en Información y Documentación (curso 2009-2010 a curso 2015 a 2016).

Fuente: elaboración propia a partir de datos del INE y de la secretaría de la Facultad de Ciencias de la Documentación de la UCM. 


\section{b.3 Número de profesores, categoría y evolución}

En el curso 2015-2016 el Grado en Información y Documentación contaba con 55 profesores procedentes de diferentes departamentos de la $\mathrm{UCM}^{4}$. La mayoría pertenecen al Departamento de Biblioteconomía y Documentación de esta Facultad. Otros departamentos que han proporcionado profesores han sido el departamento de Ciencias y Técnicas Historiográficas y Arqueología de la Facultad de Geografía e Historia; los departamentos de Filología Española IV, de Filología Española I (Lengua Española) y de Filología Inglesa, de la Facultad de Filología; el departamento de Sociología IV (1) y la sección departamental de Derecho administrativo, ambos de la Facultad de Ciencias de la Información; y, por último, el Departamento de Historia del Derecho y de las Instituciones, de la Facultad de Derecho. Conviene destacar que 50 de estos 55 profesores son doctores, lo que representa el 90,9\% del total.

\section{Gráfico 2}

\section{Profesores doctores del Grado en Información y Documentación de la UCM}

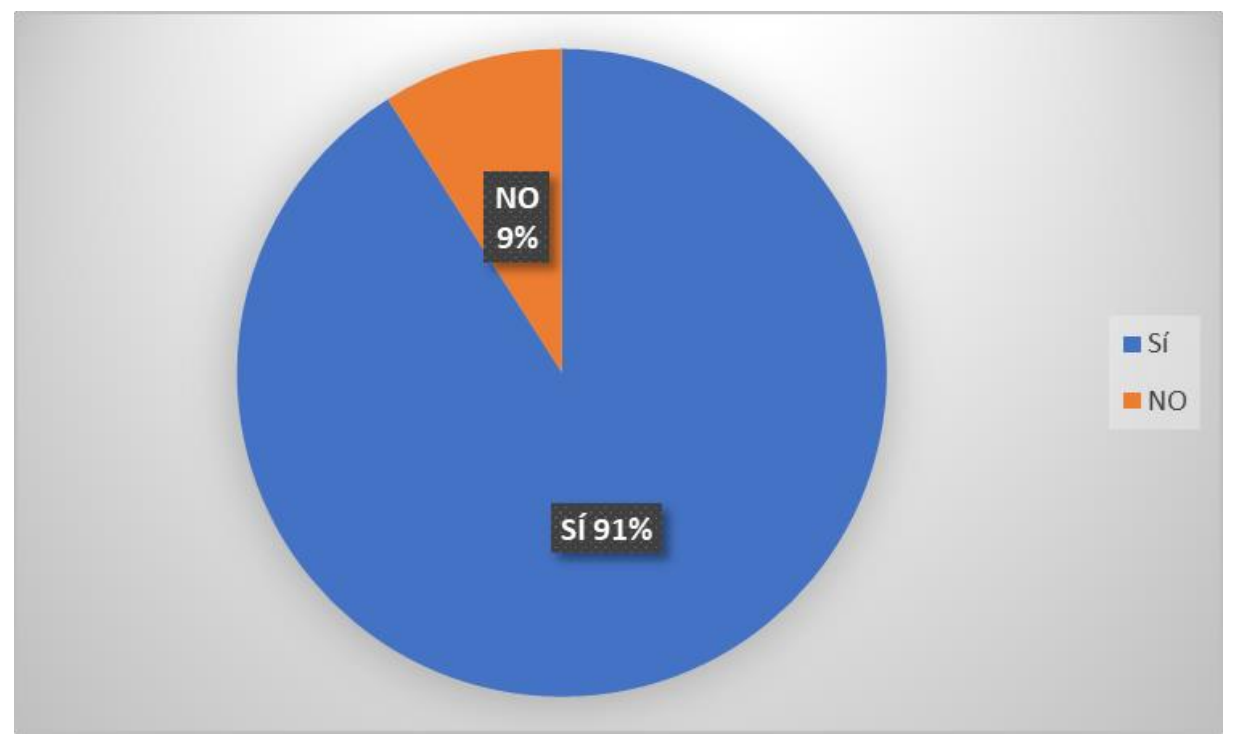

Fuente: Fundación para el Conocimiento Madri+d, 2017. 
En cuanto a su categoría, de estos 55 profesores no había ninguno que fuera catedrático de universidad; 22 eran profesores titulares de universidad (40\%); 3 eran catedráticos de escuela universitaria $(5,4 \%)$, 3 eran titulares de escuela universitaria $(5,4 \%) ; 5$ eran contratados doctores (9\%), 7 ayudantes doctores (12,7\%) y 15 eran asociados $(27,2 \%)$. En total, el porcentaje de profesorado permanente (catedráticos, titulares y contratados doctores) es de $60 \%$, frente a un $40 \%$ de no permanente (ayudantes y asociados).

\section{Gráfico 3}

\section{Categorías de los profesores del Grado en Información y Documentación de la UCM}

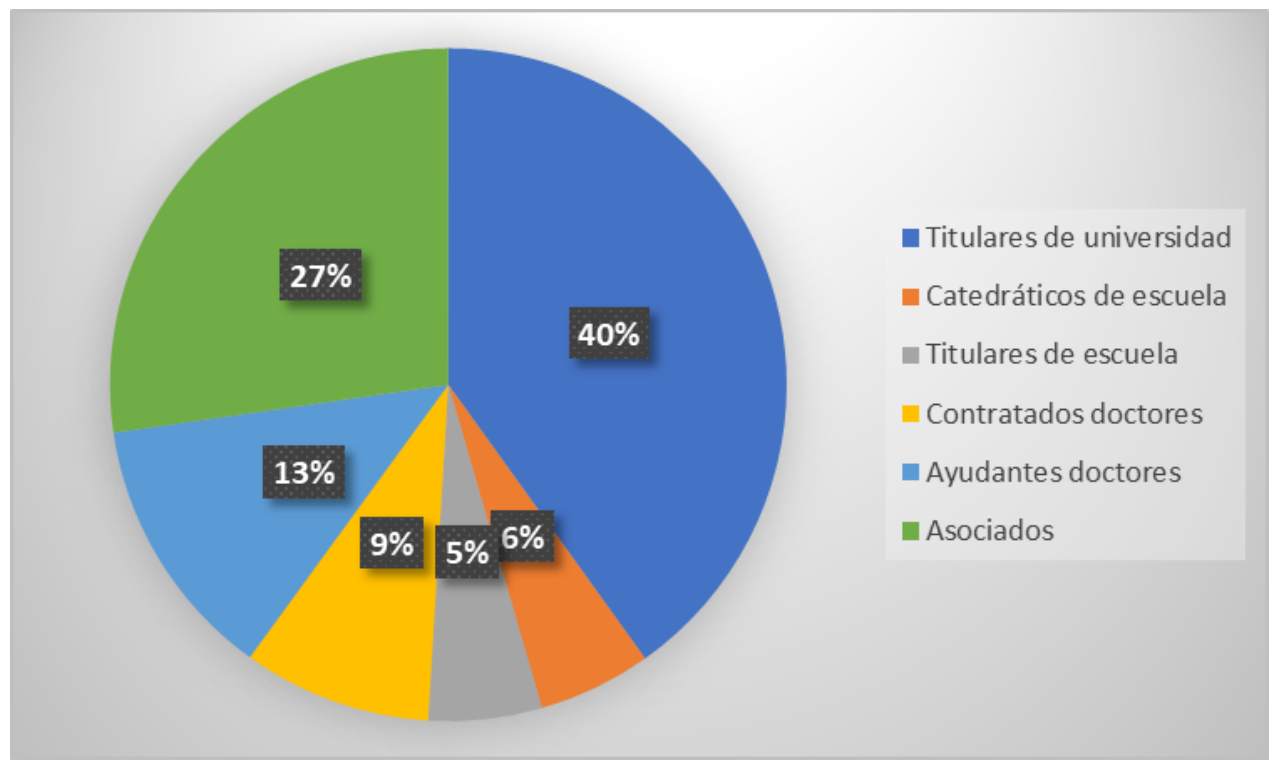

Fuente: Fundación para el Conocimiento Madri+d, 2017.

Entre el curso 2008-2009 y el 2015-2016 ha incrementado el número de profesores en cinco, ya que el primer año de impartición del grado se contaba con 50 docentes. En lo que sí ha habido mayor cambio es en sus categorías, como consecuencia de los procesos de acreditación y promoción del profesorado. Así, por ejemplo, se ha pasado de 7 titulares a 22. 


\begin{tabular}{|c|c|c|}
\hline $\begin{array}{r}\text { Cuadro } \\
\begin{array}{r}\text { Evolución de los profesores por } \\
\text { Información y Documen }\end{array}\end{array}$ & $\begin{array}{l}\text { egorías del } \\
\text { ón de la UCn }\end{array}$ & rado en \\
\hline Curso académico & $2008-2009$ & $2015-2016$ \\
\hline Catedráticos de universidad & - & - \\
\hline Titulares de universidad & 7 & 22 \\
\hline Catedráticos de Escuela universitaria & 3 & 3 \\
\hline Titulares de Escuela Universitaria & 9 & 3 \\
\hline Contratados Doctores & 5 & 5 \\
\hline Asociados & 18 & 15 \\
\hline Ayudantes Doctores & 4 & 7 \\
\hline Ayudantes & 4 & - \\
\hline Visitantes & & - \\
\hline Emérito & & - \\
\hline
\end{tabular}

Fuente: Fundación para el Conocimiento Madri+d, 2017.

\section{b.4 Las metodologías docentes del profesorado}

En relación a las tecnologías en la docencia, el profesor de esta Facultad, Pedro Razquín realizó una investigación (Razquín, 2017) sobre el uso del campus virtual basada en cuestionario aplicado a todo el profesorado y que obtuvo una respuesta del 46,6\% (28 respuestas). Los resultados más significativos son los que se recogen a continuación:

- En el campus virtual (Moodle) la mayoría de los profesores (más de 8 de cada 10) utilizan los formatos pdf y powerpoint. También los vídeos tienen una utilización considerable $(57,1 \%)$.

- Hay pocos profesores (el $10 \%$ ) que utilizan otros formatos más innovadores (Camtasia...)

- Solo el 35\% profesores evalúa la calidad de los materiales que desarrolla.

- Las herramientas que más se utilizan de Moodle son el correo, el cronograma, los foros y las tareas. En cambio otras funcionalidades como los exámenes, los trabajos en grupo o el chat son poco utilizados. 
Es importante indicar que tanto la Universidad como la propia Facultad convocan por cada curso unas ayudas económicas a proyectos de innovación educativa. Así, en el curso 20152016 la UCM concedió 7 proyectos de innovación docente vinculados al Grado en Información y Documentación. En el caso de los proyectos de la convocatoria de la Facultad fueron tres los concedidos y se tuvo en cuenta en su concesión que se tratara de un proyecto de innovación docente UCM, que sus miembros colaboraran en la implementación de proyectos que puedan ser aplicados en varios módulos o especialidades, que el proyecto se adecuara a una asignatura o grupo de asignaturas al que fuera dirigido, que se incluyera la participación del alumnado y que la duración fuera de un curso académico.

\section{b.5 La formación del profesorado}

En cuanto a la formación del profesorado, hay que señalar que la Facultad organiza todos los años los cursos relativos al campus virtual. Por otra parte, la UCM tiene un Plan de Formación del Profesorado ${ }^{5}$ con los siguientes objetivos:

- Impulsar la formación permanente del profesorado como una actividad inherente a la profesión docente e investigadora.

- Mejorar la calidad de la docencia, mediante la adquisición de habilidades, competencias y metodologías docentes.

- Estimular la innovación, la formación grupal y el intercambio de experiencias en el aula.

- Dotar al profesorado de herramientas y técnicas que le permitan impulsar su actividad investigadora y carrera académica.

Los cursos que se imparten son de las siguientes áreas:

- Área de internacionalización de la docencia y la investigación.

- Área de tecnologías para la docencia e investigación.

- Área de investigación.

- Área documental y bibliográfica.

- Área de gestión, desarrollo y consolidación profesional.

- Área de herramientas y estrategias didácticas.

- Área de conocimientos y habilidades transversales. 


\section{b.6 La investigación del profesorado}

El profesorado del Grado está involucrado en siete grupos de investigación de la propia universidad $^{6}$ que se señalan a continuación:

- BIBLIOPEGIA: Encuadernación y libro antiguo.

- BISOC: Biblioteca y Sociedad.

- FOTODOC: Fotografía y Documentación.

- NUMISDOC: Numismática e investigación documental y epigrafía.

- POLITECOM: Políticas de información, tecnologías de la Documentación y comunicación científica.

- PUBLIDOC: Gestión de la Documentación en el sector público.

- SCRINIUM: Grupo de Investigación en Archivos y Patrimonio Documental.

Analizando los profesores de la Facultad que participan en estos grupos a la fecha de agosto de 2017, se puede señalar que, de los 49 profesores adscritos a la Facultad de Ciencias de la Documentación, 33 pertenecen a algún grupo de investigación (67,3\%). Aunque a priori se podría pensar que los profesores no adscritos a ningún grupo serían los profesores asociados, el análisis indica que no hay relación entre los grupos y la categoría. Así de los 16 profesores que no pertenecen a ningún grupo hay 8 profesores permanentes $y$, sin embargo, hay 5 profesores asociados que sí forman parte de estos grupos. Señalar también que hay 4 profesores que pertenecen a dos grupos de investigación.

Para conocer las publicaciones del profesorado la mejor fuente de la que cuenta la UCM es el recién inaugurado Portal Bibliométrico de la $\mathrm{UCM}^{7}$. Esta herramienta permite conocer las publicaciones referenciadas en Google Scholar y en Scopus del personal docente e investigador de esta universidad desde el año 1980 hasta la actualidad, permitiendo agrupar este personal por Grupos de investigación, Departamentos y Facultades. Así para este periodo (1980-2017) el profesorado de la Facultad de Ciencias de la Documentación tuvo 1728 publicaciones referenciadas en Google Scholar y 153 en Scopus. 
Para saber si todos los profesores publican o solo un porcentaje de ellos, se han analizado las 27 publicaciones referenciadas en Scopus de los años 2015 y 2016. De los 49 profesores de la Facultad, 20 han sido autores de algunas de esas publicaciones (el 40\%). Con una sola publicación nos encontramos 8 profesores, con 2 publicaciones 7 profesores, con 3 publicaciones 4 profesores y con 4 publicaciones 1 profesor. Todo ello parece indicar que los profesores de la Facultad de Ciencias de la Documentación no tienen un índice elevado de publicación. Además, mientras que algunos profesores publican mucho, otros no lo hacen.

\section{Gráfico 4}

Publicaciones en Google Scholar del profesorado de la Facultad de Ciencias de la UCM

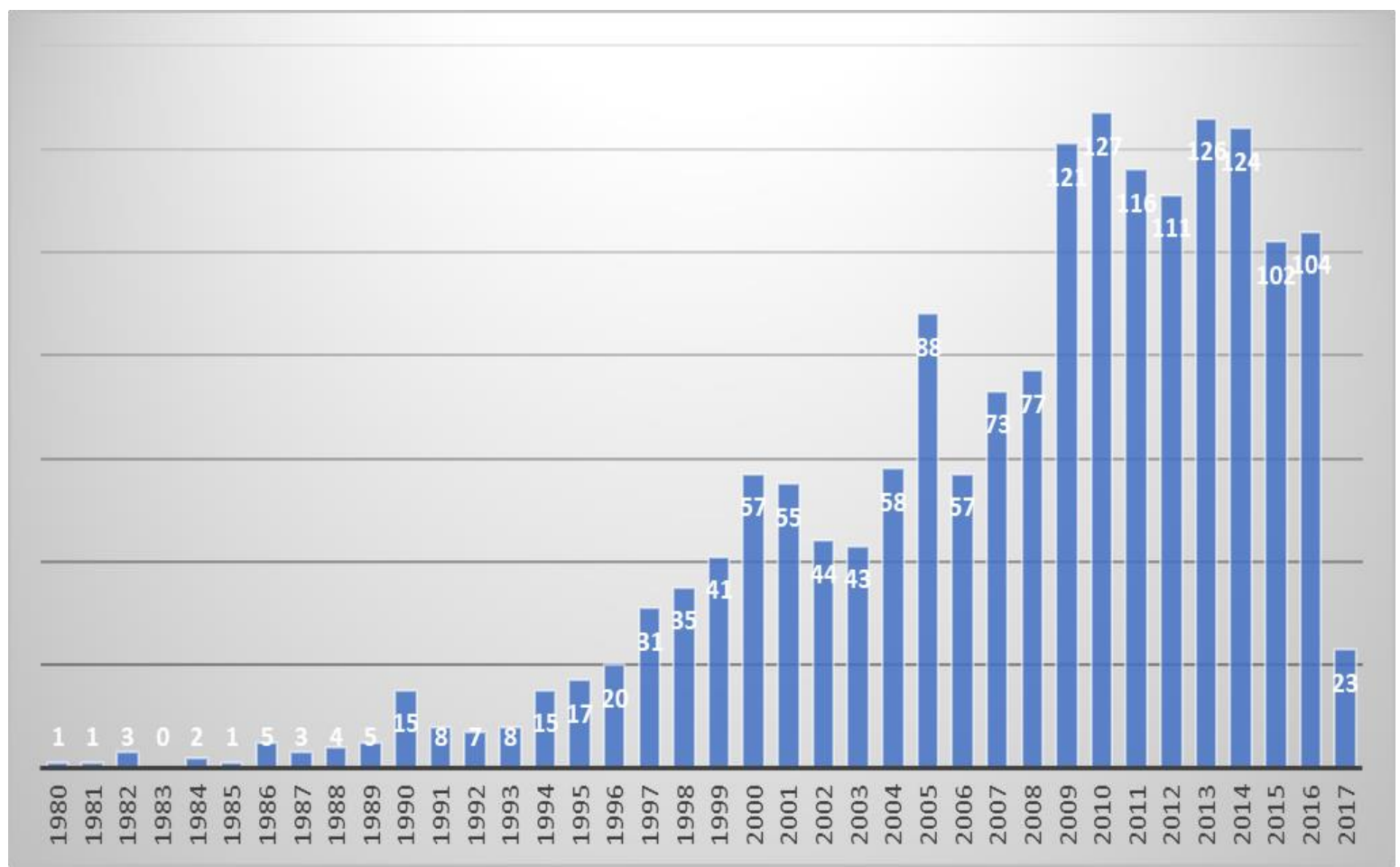

Fuente: Portal Bibliométrico UCM. 


\section{Gráfico 5}

Publicaciones en Scopus del profesorado de la Facultad de Ciencias de la UCM

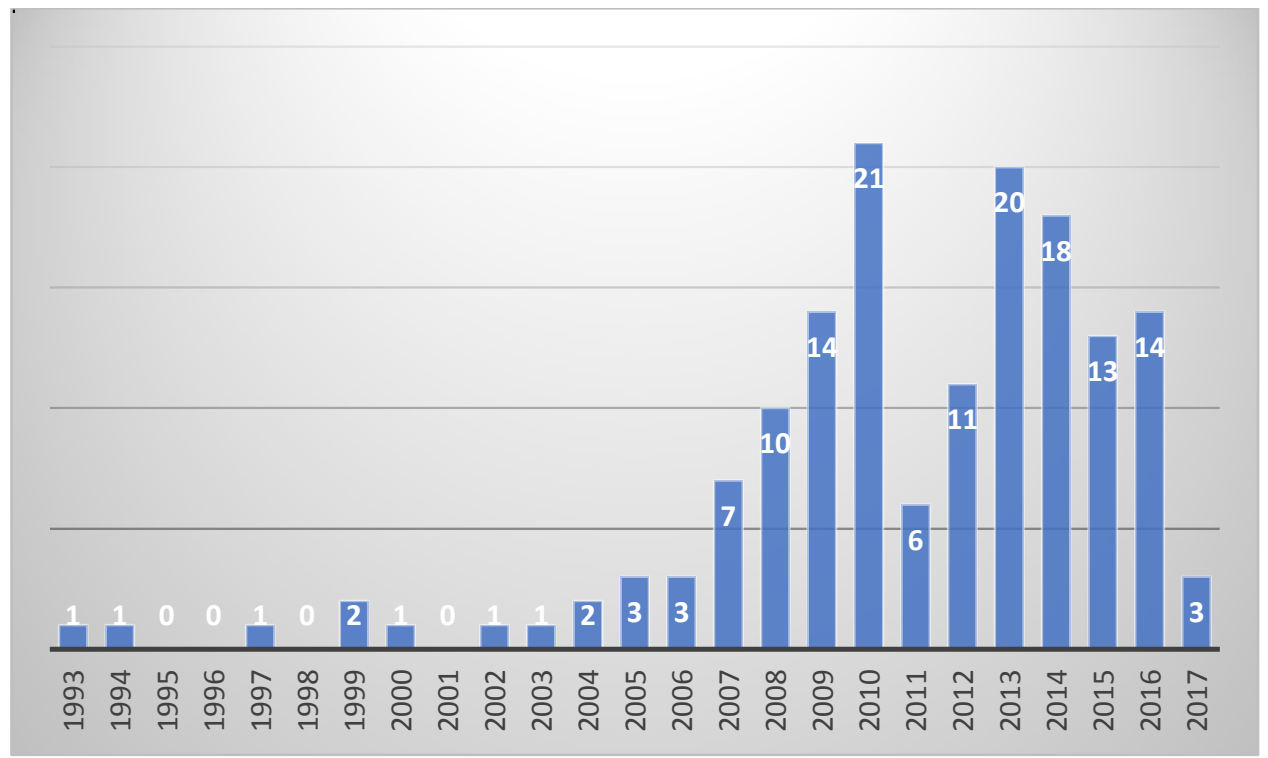

Fuente: Portal Bibliométrico, UCM.

\section{b.7 La evaluación del profesorado}

La Agencia Nacional de Evaluación de la Calidad y Acreditación (ANECA) en 1987 estableció el Programa de Apoyo a la Evaluación de la Actividad Docente del Profesorado Universitario (DOCENTIA) "con el objeto de apoyar a las universidades en el diseño de mecanismos propios para gestionar la calidad de la actividad docente del profesorado universitario y favorecer su desarrollo y reconocimiento"8.

La Universidad Complutense de Madrid a partir de este marco ha desarrollado el Programa DOCENTIA-UCM. Este "pretende reconocer la importancia de la actividad docente del profesorado en el conjunto de las actividades que realiza el mismo dentro de la UCM. Trata por tanto de ser un modelo orientado al reconocimiento de las buenas prácticas y a la mejora de la docencia, y favorecer la cultura de la calidad alineando la actividad docente del profesorado con los objetivos de la institución"'. 
Por medio de este programa se evalúa la actividad docente de cada profesor en cuatro dimensiones: planificación y organización, desarrollo, resultados y procesos de reflexión, mejora y actualización de la actividad docente.

Todos los profesores, a excepción de los asociados, tienen la obligación de someterse a esta evaluación docente que se realizará cada tres cursos académicos. Si el docente obtiene una evaluación positiva no tendrá que someterse a una nueva evaluación hasta que transcurran tres nuevos cursos académicos desde el último en que haya sido evaluado.

Esta evaluación se hace a partir de un autoinforme que cumplimenta el profesor, aportando información relativa a su participación en actividades de innovación y actualización docente. Figuran también los datos que poseen el Departamento y su Facultad sobre la participación y colaboración en las actividades de planificación y coordinación docente que son indicadores de la contribución del profesor al normal funcionamiento de la actividad docente. Además, se tiene en cuenta las encuestas anuales que cumplimentan los estudiantes sobre su actividad docente.

La calificación que obtiene el profesor es una de las cuatro categorías siguientes: excelente, muy positiva, positiva, no positiva. Entre las consecuencias de esta evaluación para el profesorado figura la Concesión de "Tramo DOCENTIA-UCM" al profesorado, evaluado con dos pruebas calificadas al menos como "positivas" en un periodo de seis años. Este tramo significa el reconocimiento formal y administrativo y se tiene en cuenta en los planes de promoción o estabilización del profesorado, en la renovación de contratos, en las convocatorias UCM de becas de movilidad o proyectos de innovación docente, y en incentivos económicos tales como las retribuciones variables ligadas a méritos individuales de la Comunidad de Madrid.

La Facultad de Ciencias de la Documentación publica en su web un informe global de resultados de la evaluación de su profesorado (Oficina Calidad UCM, 2017). Los resultados que ofrece para la convocatoria 2015-2016 son los siguientes: 
- El número de profesores evaluados fue de 32. Hay que tener en cuenta, como se ha señalado, que los profesores asociados no tienen la obligación de someterse a esta evaluación.

- La puntuación media obtenida ha sido de 85,12 puntos sobre 100 , obteniendo 68,41 puntos el profesor que menos puntación ha obtenido y 98,96 el que más (desviación típica 7,32).

- Por categorías: 11 profesores (34,38\%) han obtenido la Evaluación excelente y 21 $(65,63 \%)$ la Evaluación muy positiva. Ninguno ha sido calificado con la Evaluación positiva o la evaluación no positiva.

El cuestionario para los alumnos en el que evalúa la actividad docente del profesor consta de una serie de preguntas sobre sus datos sociodemográficos (sexo, edad y si trabajan); el seguimiento que realizan de la asignatura (horas semanales que dedican fuera de clase a preparar la asignatura y su asistencia a clase y tutorías); y la valoración subjetiva del profesor. Estas últimas preguntas son las siguientes:

1. El profesor informa de manera clara sobre los objetivos de la asignatura.

2. El profesor informa de manera clara sobre el sistema de evaluación.

3. El profesor informa de manera clara sobre las actividades docentes: trabajos, seminarios, visitas, trabajos de campo, laboratorios.

4. La bibliografía recomendada es útil para cursar la asignatura.

5. El profesor organiza y estructura bien las clases.

6. El profesor utiliza el Campus Virtual como herramienta de aprendizaje.

7. El profesor explica de forma clara y comprensible.

8. El sistema de evaluación permite al estudiante reflejar los conocimientos y competencias adquiridas.

9. El profesor cumple con el programa de la asignatura.

10. El profesor cumple con los horarios de clase establecidos.

11. El profesor cumple con el sistema de tutorías.

12. El profesor cumple con el sistema de evaluación sobre el que ha informado.

13. El profesor se muestra accesible con los estudiantes. 
14. El profesor mantiene un trato correcto con los estudiantes.

15. El profesor despierta mi interés por la asignatura.

16. La labor docente de este profesor me ayuda a adquirir conocimientos y competencias.

17. En mi opinión es un buen profesor.

18. Estoy satisfecho/a con la labor docente del profesor.

Esta evaluación al profesorado, aunque da resultados indicativos sobre el desarrollo de la docencia, presenta algunos problemas que merecen su consideración y que también, en algunos casos, han sido señalados por otros autores (Calderón, González, 2009; De Juan, Pérez-Cañaveras, 2006):

- El número de estudiantes que cursan cada asignatura por lo general es muy bajo y esta circunstancia favorece las respuestas positivas. Además, los alumnos introducen su correo electrónico para autentificarse en el sistema y aunque las encuestas son anónimas, tienen cierta creencia en que el profesor puede conocer su valoración.

- El cuestionario es el mismo para todas las titulaciones de la UCM por lo que no se tienen en cuenta las especificidades de la enseñanza en Información y Documentación ni de sus diferentes asignaturas.

- Las preguntas sobre la metodología docente reflejan sobre todo una enseñanza tradicional, basada en la clase magistral, y no hay ninguna que recoja la innovación docente del profesor en el contexto de su asignatura.

- La encuesta está sobre todo orientada en saber la satisfacción del alumno dejando de lado otras cuestiones de importancia fundamental en el desarrollo de la docencia.

- Uno de los indicadores que se tienen para la obtención de la evaluación como excelente es que todos los estudiantes de la asignatura obtengan buenas calificaciones. Esto está provocando que algunos profesores evalúen injustamente a sus alumnos, sobrevalorando sus calificaciones, para así obtener la mayor categoría en su evaluación. 


\section{Conclusiones}

Se ha realizado una aproximación al perfil del profesorado del Grado en Información y Documentación de la UCM pudiendo establecer una serie de características:

- Para el número de alumnos que ingresan en la Facultad de Ciencias de la Documentación se puede considerar que la cantidad de profesores que existe es adecuado.

- Un rasgo positivo es que la mayoría de estos profesores son doctores, el 90,9\%.

- También es apropiada su distribución en categorías, ya que más de la mitad, el $60 \%$, son profesores permanentes; es decir, que o bien son funcionarios o bien tienen contratos indefinidos con la Universidad.

- La mayoría del profesorado de la Facultad (el 67,3\%) pertenece a algún grupo de investigación de la propia universidad, porcentaje también elevado.

- El número de publicaciones del profesorado de la Facultad no es muy elevado, así tan solo el $40 \%$ de ellos ha publicado en revistas referenciadas en Scopus en los dos últimos años.

- Los datos que ofrece la evaluación realizada en el curso 2015-2016 por el Programa Docentia-UCM a los docentes de la Facultad son muy positivos, sin embargo, hay que realizar algunas matizaciones sobre las características de esta valoración.

\section{Referencias bibliográficas}

Alfaro, I.; Pérez, A. (2011). Un estudio acerca de la dedicación docente del profesorado universitario en el marco del Espacio Europeo de Educación Superior. Revista interuniversitaria de formación del profesorado, 14 (4). Disponible en: http://www.aufop.com/aufop/uploaded_files/articulos/1327436198.pdf

ANECA (Agencia Nacional de Evaluación de la Calidad y Acreditación). (2003). Programa de Convergencia Europea. El crédito europeo. Disponible en http://www.aneca.es/publicaciones/docs/publi_credito\%20europeo.pdf 
Calderón, C.; González, A. (2009). El papel del profesor universitario español en el EEEs. II Congreso Internacional X Seminario Iberioamericano Motiva La Universidad y elemprendimiento, 27-30 octubre 2009, Valencia. Disponible en: http://www.uv.es/motiva2/Ponencias\%20Motiva2009/docs/53.pdf

De Juan, J.; Pérez-Cañaveras, R.M. (2006). Reflexiones en torno a la evaluación del profesorado. ¿Vigilar y Castigar? IV Jornadas de Redes de Investigación en Docencia Universitaria. ICE, Universidad de Alicante; Alicante, 5 y 6 de junio de 2006.

Fundación para el Conocimiento Madri+d. (2017). Informe de Autoevaluación Grado en Información y Documentación. UCM. 2500915/2015-2016 [Documento interno de la Facultad de Ciencias de la Documentación para la acreditación de su grado]

Madrid, J. M. (2005) La formación y la evaluación docente del profesorado universitario ante el espacio europeo de educación superior. En Educatio, n.23. Disponible en: http://hdl.handle.net/10201/26744

Oficina para la calidad UCM. (2017). Informe global de resultados por centros: Facultad de CC. de la Documentación. Programa DOCENTIA. Convocatoria 2015/16. Disponible en: https://www.ucm.es/data/cont/docs/1158-2017-07-03-Informe\%20global\%20Docentia\%2020152016.Facultad\%20de\%20Ciencias\%20de\%20la\%20Documentaci\%C3\%B3n.pdf

Razquín, P. (2017). Uso de las TIC en la Facultad de Ciencias de la Documentación: Un análisis de las prácticas docentes [Presentación de distribución interna de la Facultad de Ciencias de la Documentación de la UCM].

Valcárcel, M. (2004). La preparación del profesorado universitario para la convergencia europea en educación superior. Disponible en: http://www.univ.mecd.es/univ/html/ informes/estudios_analisis/resultados_2003/EA2003_0040/informe_final.pdf

Valcárcel, M. (2006). Informe final del estudio Reflexión sobre el proceso de Transición hacia el EEES en las universidades españolas. Disponible en: http://www.francescesteve.es/eportfolio/publicaciones/Informe-EEES.pdf 


\section{Notas del autor}

* Carlos Miguel Tejada Artigas: Doctor en Documentación. Profesor titular de la Facultad de Ciencias de la Documentación de la Universidad Complutense de Madrid. España. Correo electrónico cmtejada@ucm.es

${ }^{1}$ El texto completo puede consultarse en:

http://www.mec.es/univ/html/informes/EEES 2003/Declaracion Sorbona.pdf

${ }^{2}$ El texto completo puede consultarse en:

http://www.mec.es/univ/html/informes/EEES 2003/Declaracion Bolonia.pdf

${ }^{3}$ Más información: http://www.ucm.es/informacionydocumentacion/

${ }^{4}$ Para los datos de este apartado se ha consultado documentación de la Facultad de Ciencias de la Documentación para los procesos de acreditación del Grado.

${ }^{5}$ http://cfp.ucm.es/formacionprofesorado/

${ }^{6}$ http://documentacion.ucm.es/grupos

${ }^{7}$ https://bibliometria.ucm.es/inicio

${ }^{8}$ http://www.aneca.es/Programas-de-evaluacion/DOCENTIA

${ }^{9}$ https://www.ucm.es/programadocentia 